\title{
Transient reduction in macular deep capillary density on optical coherence tomography angiography after phacoemulsification surgery in diabetic patients
}

Zaowen Wang ${ }^{1,2+}$, Erqian Wang ${ }^{1,2+}$ and Youxin Chen ${ }^{1,2^{*}}$ (D)

\begin{abstract}
Background: To evaluate macular microvascular changes and associated factors in diabetic patients following uncomplicated phacoemulsification surgery.

Methods: In this prospective observational study, we enrolled diabetic patients and non-diabetic controls who underwent phacoemulsification surgery. Participants were examined at postoperative day 1 (POD1), 10 (POD10), 30 (POD30), and 90 (POD90), using macular 3×3mm OCT angiography scan (RTVue-XR Avanti; Optovue, Inc., Fremont, (A). Integrated automated algorithms were used to quantify parafoveal vessel density (VD) in superficial capillary plexus (SCP) and deep capillary plexus (DCP). To minimize measurement bias, subjects with corneal edema or capsular opacity at any postoperative visit were excluded.

Results: The study included 21 eyes of 21 diabetic patients and 21 eyes of 21 non-diabetic controls. In diabetic patients, no significant change in SCP-VD could be detected ( $P=0.57$ ); DCP-VD reduced from $50.24 \pm 2.33 \%$ at POD1 to $48.33 \pm 3.07 \%$ at POD30 $(P=0.019)$, and restored to $50.74 \pm 3.44 \%$ at POD90 $(P=1.00)$. The DCP-VD change at POD30 in diabetic patients $(-1.90 \pm 2.61 \%)$ was significantly different from that in controls $(1.31 \pm 2.61 \%)(P<$ 0.001). The amount of DCP-VD reduction was correlated with foveal and parafoveal thickening $(r=0.431, P=0.051$ and $r=0.514, P=0.017$, respectively), high cumulative dissipated energy $(P=0.032)$ and increased hemoglobin A1c concentration $(P=0.037)$.

Conclusions: Phacoemulsification in diabetic patients caused transient reduction in DCP-VD, which was associated with poor glycemic control, surgical trauma, and postoperative macular thickening. Our results added a new dimension to our understanding of the complex biologic effects of cataract surgery in diabetic subjects.
\end{abstract}

Keywords: Phacoemulsification surgery, Macular edema, Optical coherence tomography angiography, Vessel density, Diabetes

\footnotetext{
* Correspondence: chenyx@pumch.cn

'Zaowen Wang and Erqian Wang contributed equally to this work.

'Department of Ophthalmology, Peking Union Medical College Hospital, Beijing 100730, China

${ }^{2}$ Key Laboratory of Ocular Fundus Diseases, Chinese Academy of Medical

Sciences, Beijing 100730, China
}

C C The Author(s). 2020 Open Access This article is licensed under a Creative Commons Attribution 4.0 International License, which permits use, sharing, adaptation, distribution and reproduction in any medium or format, as long as you give appropriate credit to the original author(s) and the source, provide a link to the Creative Commons licence, and indicate if changes were made. The images or other third party material in this article are included in the article's Creative Commons licence, unless indicated otherwise in a credit line to the material. If material is not included in the article's Creative Commons licence and your intended use is not permitted by statutory regulation or exceeds the permitted use, you will need to obtain permission directly from the copyright holder. To view a copy of this licence, visit http://creativecommons.org/licenses/by/4.0/ The Creative Commons Public Domain Dedication waiver (http://creativecommons.org/publicdomain/zero/1.0/) applies to the data made available in this article, unless otherwise stated in a credit line to the data. 


\section{Background}

Phacoemulsification cataract surgery is one of the most common anterior segment surgical procedures. Although it is now safer than before, complications are occasionally seen. Pseudophakic cystoid macular edema (PCME) is one of the important posterior segment complications with a baseline incidence rate of $1.17 \%$ [1]. Diabetes has been associated with increased incidence of PCME [1, 2], but how diabetes increases the risk remains unclear.

With the advent of optical coherence tomography angiography (OCTA), macular microvasculatures, including superficial and deep capillary plexus, can be visualized and quantified non-invasively [3, 4]. In previous studies which focused on non-diabetic subjects, cataract surgery was reported to cause an increase in macular microvasculature vessel density (VD) [5-7]. However, none of the previous studies included diabetic subjects, and how cataract surgery influence macular microvasculature networks in diabetic eyes remains unknown.

The current study focused on diabetic patients and aimed to study how their macular microvasculature networks responded to phacoemulsification surgery, compared with non-diabetic patients. We also aimed to investigate whether the microvascular changes were associated with postoperative macular thickening or baseline clinical characteristics.

\section{Methods}

The study followed the tenets of the Declaration of Helsinki. Ethics committee of Peking Union Medical College Hospital approved the study protocol. All participants gave informed consent.

\section{Patients}

Patients with type 2 diabetes undergoing phacoemulsification cataract surgery with an experienced cataract surgeon (Z.W.) between October 2018 and April 2019 were consecutively evaluated for this prospective study. We also recruited age- and lens grading-matched nondiabetic subjects as controls during the same period. Participants were excluded at baseline if they had: 1) age younger than 50 years or older than 80 years; 2) high myopia of over -6.00 diopters or over $26.50 \mathrm{~mm}$ in axial length; 3) diabetic macular edema, intraretinal microcyst or any other diabetic maculopathy at baseline; 4) retinal diseases except for mild or moderate nonproliferative diabetic retinopathy (NPDR); 5) history of retinal laser or intravitreal injection; or 6) other systemic conditions including but not limited to uncontrolled hypertension, autoimmune diseases, and malignant tumor. Eligible subjects underwent phacoemulsification surgery and were followed up at postoperative day 1 (POD1), 10 (POD10), 30 (POD30), and 90 (POD90). Subjects were further excluded for final analysis if they: 1) developed intraoperative or postoperative complication except for cystoid macular edema; 2) exhibited postoperative corneal edema or posterior capsule opacity that might interfere with accurate OCTA measurement at any postoperative visit; 3) failed to generate OCTA scan quality score of $\geq 7$ at any visit; or 4 ) were lost to followup.

Patients were evaluated by slit lamp biomicroscope before surgery for cataract grading in Lens Opacities Classification System III [8]. Patients were also evaluated by indirect ophthalmoscope via dilated pupil at POD1 for diabetic retinopathy grading based on the Early Treatment Diabetic Retinopathy Study classification system [9]. Time from diagnosis of diabetes, glycemic control measured as hemoglobin A1c (HbA1c) concentration, and insulin dependence were recorded. HbA1c was tested with commercially available kit (Bio-Rad Laboratories, Inc., Hercules, CA).

\section{Surgical technique}

All surgeries were performed by one surgeon (Z.W.) using Centurion Vision System (Alcon Laboratories, Inc., Texas, USA) under topical anesthesia. Briefly, after a $2.4 \mathrm{~mm}$ clear corneal incision was made, continuous capsulorhexis, hydrodissection, intracapsular phacoemulsification cataract extraction, irrigation/aspiration cortex removal, and posterior capsule polish were sequentially performed. Foldable hydrophilic acrylic intraocular lens (Akreos MI60, Bausch \& Lomb, Rochester, N.Y., USA) was implanted in the capsular bag. Cumulative dissipated energy (CDE) and ultrasound total time (US) were recorded. Patients were administered topical diclofenac and levoflaxacin for 3 days before surgery and 4 weeks after surgery. Loteprednol etabonate eye drops were also applied and tapered for 4 weeks after surgery.

\section{Optical coherence tomography angiography image acquisition and vessel density measurement}

Optical coherence tomography angiography scans were performed on the commercially available RTVue XR Avanti spectral domain OCTA device (Optovue Inc. Fremont, CA, USA). This device uses light source of 840 $\mathrm{nm}$ wavelength and operates at $70000 \mathrm{~A}$-scans per second to generate OCTA volume scans composed of $304 \times 304$ A-scans over $3.0 \times 3.0 \mathrm{~mm}$ macular area. At each visit, three consecutive macular scans centered on fovea with scan quality score $\geq 7$ were obtained, and the one with lowest score was discarded. All image acquisitions were made by one single investigator (E.W.).

The quantitative measurements of vessel density were made by a built-in software AngioVue AngioAnalytics (Version 2017.1, Optovue Inc. Fremont, CA, USA) which uses split-spectrum amplitude decorrelation angiography algorithm with three-dimensional projection 
artifact removal technique. By default settings, foveal region is defined as the inner round area with $1.0 \mathrm{~mm}$ diameter, and parafoveal region is defined as an annulus area with inner and outer diameter of $1.0 \mathrm{~mm}$ and 3.0 $\mathrm{mm}$, respectively. Superficial capillary plexus (SCP) extended from internal limiting membrane to $10 \mu \mathrm{m}$ above inner plexiform layer. Deep capillary plexus (DCP) extended from $10 \mu \mathrm{m}$ above inner plexiform layer to $10 \mu \mathrm{m}$ below outer plexiform layer. The boundaries of capillary plexus were automatically recognized by the software and independently reviewed by two authors (E.W. and Y.C.) in case that manual adjustment was needed. The software automatically generated parafoveal SCP-VD and DCP-VD. Macular thickness of foveal and parafoveal area was also provided based on the same OCTA volume scan dataset. The measurements from two scans were averaged for further analysis. We did not include preoperative OCTA measurements because cataract could result in low signal strength and inaccurate quantification of OCTA metrics $[10,11]$. The measurements at POD1 were used as baseline value for calculating VD changes and macular thickening.

\section{Statistical analysis}

Statistical analyses were performed with SPSS software version 19 (SPSS, Inc., IL, Chicago, USA). Variable normality was inspected using Kolmogorov-Smirnov test. Baseline clinical characteristics were compared using one-way ANOVA and chi-square test for continuous variables and dichotomous variables, respectively. Postoperative VD changes in diabetic patients were evaluated using repeated measures ANOVA and Bonferroni's post hoc test. The VD changes from baseline was compared between diabetic patients and controls using ANCOVA after adjusting for $\mathrm{CDE}$ and changes in scan quality score. Univariate Pearson correlation or Spearman correlation, where appropriate, was used to assess $\mathrm{SCP} /$ DCP-VD changes for their association with macular thickening and baseline clinical characteristics including age, gender, axial length, HbA1c level, duration since diagnosis of diabetes, insulin dependence, presence of NPDR at baseline, nuclear opalescence (NO); nuclear color (NC), CDE, and US. We then picked out candidate baseline characteristics for further multivariate linear regression to define the factors that significantly correlated with vessel density changes. A $P$ value of less than 0.05 was considered as statistically significant.

\section{Results}

The study enrolled 24 patients with type 2 diabetes, excluded 3 patients ( 1 lost to follow-up, 1 had OCTA scan quality score of lower than 7 at POD1 due to mild corneal edema, and 1 developed mild vitreous hemorrhage and received intravitreal injection at 6 weeks after cataract surgery due to rapid progression of diabetic retinopathy), and included 21 eyes of 21 diabetic patients for further analysis. The control group included 21 matched eyes of 21 non-diabetic subjects. At baseline, 5 of the 21 diabetic patients had mild or moderate NPDR, and the other 16 had no apparent retinopathy. At POD90, 1 of the 5 patients with NPDR at baseline developed mild cystoid macular edema at POD90. The baseline demographic characteristics and OCTA scan quality score at all follow-up visits are comparable between diabetic and control groups (Table 1).

\section{Macular vessel density changes}

In patients with diabetes, no significant change in SCP$\mathrm{VD}$ could be detected among the four postoperative visits $(P=0.57)$. A transient reduction in DCP-VD was noted at POD30 $(P=0.019)$ which restored to baseline level at POD90 $(P=1.00)$. A continuous increase in foveal and parafoveal thickness was also observed after surgery (both $P<0.001)$. The longitudinal changes of SCP-VD, DCP$\mathrm{VD}$, foveal thickness, and parafoveal thickness in diabetic patients and controls are shown in Fig. 1.

Comparison between diabetic patients and nondiabetic controls revealed no statistically significant difference in SCP-VD changes. However, the DCP-VD change at POD30 in diabetic patients $(-1.90 \pm 2.61 \%)$ was significantly different from that in controls $(1.31 \pm$

Table 1 Demographics and OCTA scan quality score of diabetic and control Subjects

\begin{tabular}{|c|c|c|c|}
\hline Characteristics & $\begin{array}{l}\text { Diabetics } \\
(n=21)\end{array}$ & $\begin{array}{l}\text { Controls } \\
(n=21)\end{array}$ & $P$ values \\
\hline Age (years) & $70.0 \pm 8.3$ & $70.6 \pm 6.0$ & $0.80^{*}$ \\
\hline Gender (M:F) & 11:10 & $8: 13$ & $0.35^{\dagger}$ \\
\hline Axial Length (mm) & $23.32 \pm 1.30$ & $23.72 \pm 0.93$ & $0.26^{*}$ \\
\hline Duration of diabetes (years) & $15.1 \pm 8.9$ & NA & NA \\
\hline $\mathrm{HbA1c}(\%)$ & $7.31 \pm 1.00$ & NA & NA \\
\hline Insulin dependence & $11(52.4 \%)$ & NA & NA \\
\hline NO & $2.86 \pm 0.69$ & $2.79 \pm 0.57$ & $0.70^{*}$ \\
\hline NC & $2.97 \pm 0.71$ & $2.87 \pm 0.59$ & $0.64^{*}$ \\
\hline CDE (second) & $5.16 \pm 1.59$ & $4.65 \pm 1.53$ & $0.30^{*}$ \\
\hline US (second) & $56.3 \pm 11.8$ & $51.7 \pm 10.9$ & $0.19^{*}$ \\
\hline \multicolumn{4}{|l|}{ OCTA scan quality score } \\
\hline POD1 & $8.05 \pm 0.55$ & $8.12 \pm 0.61$ & $0.69^{*}$ \\
\hline POD10 & $8.10 \pm 0.62$ & $8.29 \pm 0.60$ & $0.32^{*}$ \\
\hline POD30 & $8.14 \pm 0.74$ & $8.14 \pm 0.67$ & $1.00^{*}$ \\
\hline POD90 & $8.14 \pm 0.65$ & $8.33 \pm 0.56$ & $0.32^{*}$ \\
\hline
\end{tabular}

Data are shown as ratio, No. (\%), or mean \pm standard deviation

${ }^{*} P$ values are from one-way ANOVA

${ }^{\dagger} P$ values are from Pearson Chi-square test

NO nuclear opalescence; NC nuclear color; $C D E$ cumulative dissipated energy US ultrasound total time; NA not applicable 


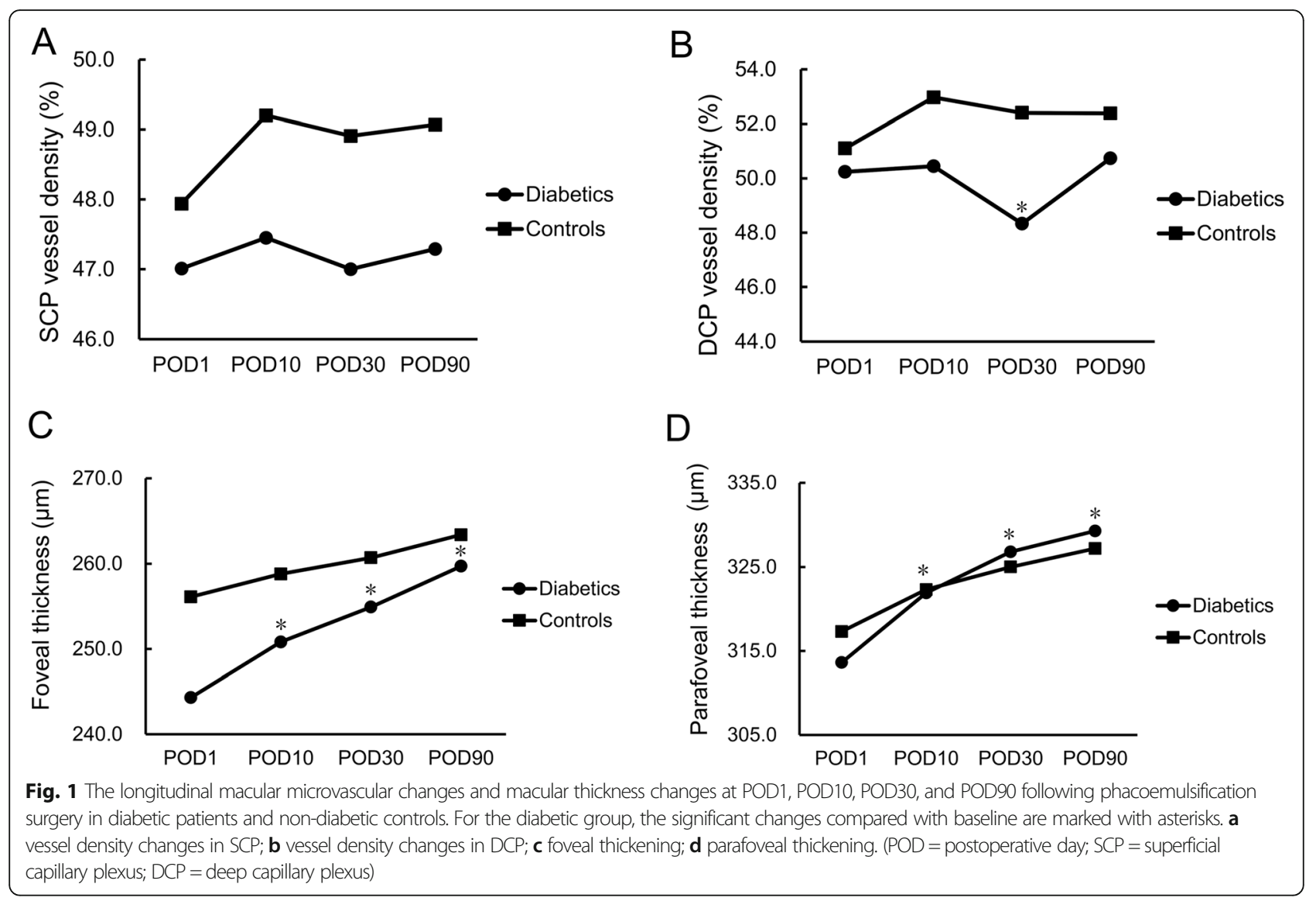

2.61\%) after adjusting for $\mathrm{CDE}$ and changes in scan quality score $(P<0.001)$ (Fig. 2$)$.

\section{Associated factors of macular vessel density changes}

In the diabetic group, univariate analysis indicated a correlation between DCP-VD reduction and foveal and parafoveal thickening at POD30 $(r=0.431, P=0.051$, and $r=0.514, P=0.017$, respectively). No significant correlation was found between SCP-VD changes and macular thickening (all $P>0.05$ ). In the control group, univariate analysis detected no significant correlation between vessel density changes and macular thickening (all $P>0.05$ ). Correlations between vessel density changes and macular thickness changes are shown in Fig. 3.

In the diabetic group, univariate analysis indicated that DCP-VD reduction at POD30 was significantly correlated with increased $\operatorname{CDE}(r=0.503, P=0.020)$, US $(r=$ $0.500, P=0.021)$ and HbA1c concentration $(r=0.492$, $P=0.023)$. Other factors were not significantly correlated with vessel density changes in DCP or SCP. Multivariate linear regression in the diabetic group revealed that for every increase in HbA1c of 1\%, the amount of DCP-VD reduction at POD30 would be added by $1.08 \%$ (95\% confidence interval 0.07 to $2.08 \%, P=0.037$ ). For every increase in $\mathrm{CDE}$ of $1 \mathrm{~s}$, the amount of DCP-VD reduction at POD30 would be added by $0.70 \%$ (95\% confidence interval 0.07 to $1.33 \%, P=0.032$ ). When analyzing all the diabetic and control subjects as a whole group, univariate analysis indicated that DCP-VD reduction at POD30 was significantly correlated with the presence of diabetes $(r=0.523, P<0.001)$ and possibly correlated with increased CDE $(r=0.290, P=0.062)$ and US $(r=0.295, P=$ 0.058). Multivariate linear regression in the whole group revealed that the presence of diabetes was the only independent factor influencing DCP-VD reduction at POD30 $(P<0.001)$. Neither CDE $(P=0.13)$ or US $(P=$ $0.16)$ had significant influence on DCP-VD change using multivariate model in the whole group. Correlations between vessel density changes and baseline clinical characteristics are shown in Fig. 4.

\section{Discussion}

To the best of our knowledge, the current study is the first one that uses OCTA in describing microvascular changes following uncomplicated phacoemulsification cataract surgery in diabetic patients. We revealed that phacoemulsification surgery caused a transient reduction in the DCP-VD, which could be restored to baseline level at 3 months after surgery. We also found that the degree of DCP-VD reduction was associated with 


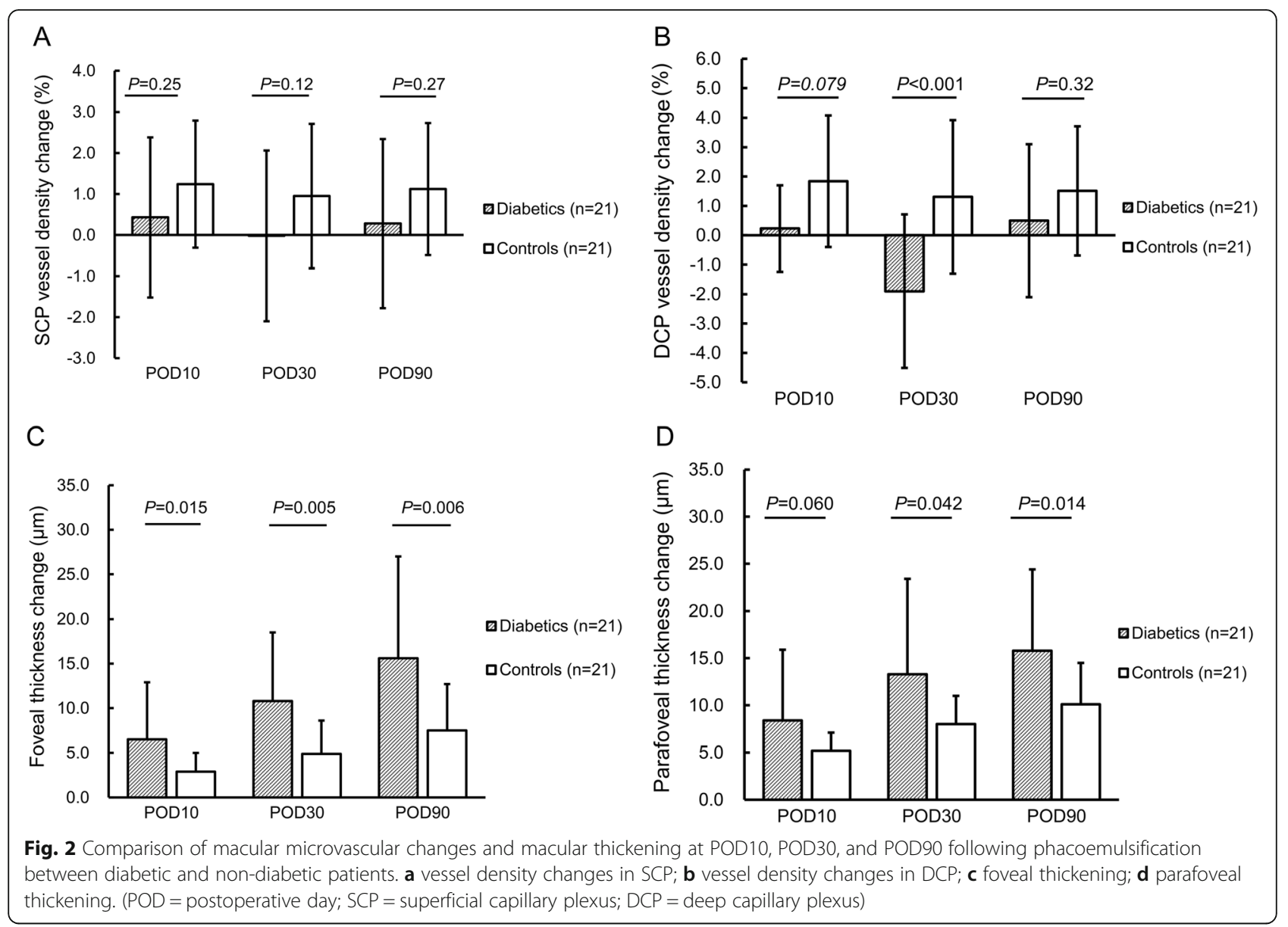

postoperative macular thickening, glycemic control, and surgical trauma.

Our findings suggested that the microvasculature networks in diabetic eyes responded differently to phacoemulsification surgery, mainly in the deep capillary plexus, compared with non-diabetic eyes. Previous studies reported a significant increase in macular vessel density in non-diabetic patients [6, 7]. Pilotto et al. [6] reported a $3-5 \%$ increase in the intermediate and deep capillary plexus following cataract surgery. In the study by Zhao et al. [7], parafoveal vessel density increased by approximately $5 \%$ at 1 month after phacoemulsification. In this study, non-diabetic controls showed mild increase in both SCP-VD and DCP-VD, however, diabetic eyes failed to show any increase in SCP-VD and even demonstrate a $1.90 \pm 2.61 \%$ decline in DCP-VD. As opposed to the subtle changes in SCP-VD, the transient reduction in DCP-VD was recognized as a key feature in the postoperative microvascular changes in diabetic eyes. One possible explanation for the preferential involvement of DCP-VD was that DCP might be more sensitive to injury and more vulnerable to surgical trauma. Histopathological studies revealed that diabetic microaneurysms mainly originated from deeper part of retinal capillary plexus [12]. A growing body of OCTA studies also supported the distinct value of monitoring early microvascular changes in DCP in diabetic patients. Chen et al. [13] indicated that the fractal dimension changes in deep retinal capillary layer could be an early indicator of microvasculature changes associated with diabetic retinopathy. The OCTA metrics in DCP was not only an indicator of diabetic retinopathy severity [14], but also a predictor of diabetic retinopathy progression [15]. Furthermore, the low skeleton density in DCP was correlated with poor vision in patients with diabetic macular edema [16]. In concordance with previous studies, our results suggested the value of OCTA metrics on DCP in assessing microvascular changes following phacoemulsification surgery in diabetic patients.

The distinct pattern of microvascular changes in diabetic patients brought us to consider whether there was an association between postoperative macular thickening and macular vessel density changes in diabetic patients, especially at the level of deep macular capillaries. Previous OCTA studies have shown that non-diabetic PCME eyes have impaired deep macular microvascular density $[17,18]$. In the study by Chetrit et al. [18], PCME eyes had significantly lower vessel density in DCP (44.1 \pm 


\begin{tabular}{|c|c|c|c|c|}
\hline$A$ & ${ }_{5} C^{p}-V D$ & $\begin{array}{l}n g^{e^{s}} \\
c^{c^{p}-V}\end{array}$ & & \multirow{2}{*}{$\begin{array}{l}\text { correlation } \\
1.0\end{array}$} \\
\hline Foveal thickening & 0.24 & -0.21 & \multirow{2}{*}{ POD10 } & \\
\hline Parafoveal thickening & 0.12 & -0.30 & & 0.5 \\
\hline Foveal thickening & -0.07 & -0.43 & & \\
\hline Parafoveal thickening & -0.28 & -0.51 & POD30 & \\
\hline Foveal thickening & -0.03 & -0.18 & חרקסP & \\
\hline rafoveal thickening & 0.05 & -0.26 & & \\
\hline
\end{tabular}

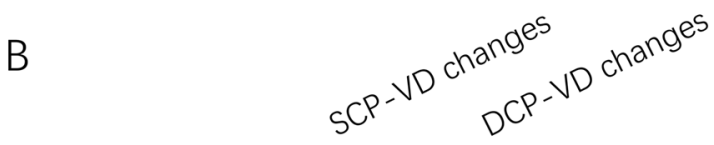

\begin{tabular}{|c|c|c|c|}
\hline & & & \\
\hline Foveal thickening & 0.22 & 0.07 & \\
\hline Parafoveal thickening & 0.24 & 0.12 & PODIO \\
\hline Foveal thickening & 0.04 & -0.06 & \\
\hline Parafoveal thickening & 0.11 & 0.00 & PODSO \\
\hline Foveal thickening & 0.35 & 0.28 & Pחר \\
\hline Parafoveal thickening & 0.21 & 0.08 & \\
\hline
\end{tabular}

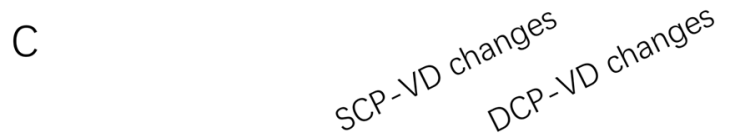

\begin{tabular}{|c|c|c|c|}
\hline & & Du. & \multirow{3}{*}{ POD10 } \\
\hline Foveal thickening & 0.11 & -0.23 & \\
\hline Parafoveal thickening & 0.06 & -0.27 & \\
\hline Foveal thickening & -0.14 & -0.46 & \multirow{2}{*}{ POD30 } \\
\hline Parafoveal thickening & -0.25 & -0.46 & \\
\hline Foveal thickening & -0.04 & -0.13 & \multirow{2}{*}{ POD90 } \\
\hline Parafoveal thickening & -0.01 & -0.22 & \\
\hline
\end{tabular}

Fig. 3 The correlation matrix plot for the association between macular thickening and vessel density changes in (a) diabetic patients ( $n=21)$, b non-diabetic controls $(n=21)$, and $(\mathbf{c})$ all study subjects $(n=42)$. Statistically significant correlations are shown in bold. (POD = postoperative day; $\mathrm{SCP}=$ superficial capillary plexus; $\mathrm{DCP}=$ deep capillary plexus; $\mathrm{VD}=$ vessel density)

$7.4 \%)$ than in controls $(54.2 \pm 3.2 \%)$, which could be restored $(51.3 \pm 6.1 \%)$ after resolution of edema. In another study by Sacconi et al. [17], PCME eyes had reduced vessel density in DCP which did not recover after treatment. Quantitative OCTA studies in diabetic patients have also identified reduced DCP flow in diabetic macular edema $[16,19]$. In this study, macular thickness changes were analyzed, and our results were in line with prior structural OCT studies that diabetic patients had more pronounced macular thickening after cataract surgery compared with non-diabetic controls [20]. Moreover, this study detected a significant correlation between macular thickening and DCP-VD reduction. Our study not only supported, but also added new longitudinal evidence to previous cross-sectional observations that macular microvascular changes are involved in the complex pathophysiological process of macular thickening following phacoemulsification surgery.

It is noteworthy that the degree of DCP-VD reduction was only $1.90 \pm 2.61 \%$ after adjusting for covariates, not as large as previously reported in PCME subjects [18]. Several reasons accounted for the mild reduction in DCP-VD in our study. First, only one of the 21 study subjects developed PCME, and the other 20 subjects had 


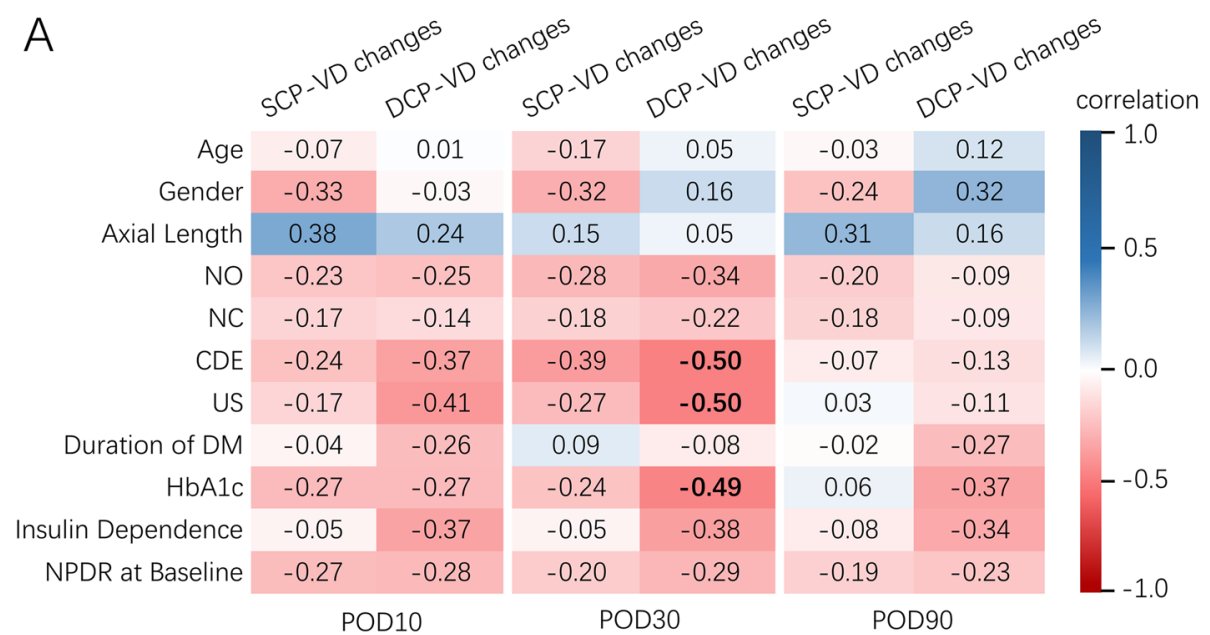

B

\begin{tabular}{|c|c|c|c|c|c|c|c|}
\hline & $c p-V D$ & $\begin{array}{l}a^{n^{e s}} \\
{ }_{D} P^{-V}-V D\end{array}$ & 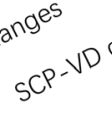 & $a^{a n e^{e s}}$ & 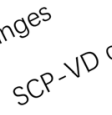 & $\begin{array}{l}a_{n} g^{e^{s}} \\
D^{C P}-V D\end{array}$ & \multirow{2}{*}{$\begin{array}{c}\text { correlation } \\
1.0\end{array}$} \\
\hline Age & -0.01 & -0.40 & -0.10 & -0.57 & -0.28 & -0.18 & \\
\hline Gender & 0.11 & -0.08 & -0.04 & -0.17 & 0.03 & -0.32 & 0.5 \\
\hline Axial Length & 0.73 & 0.52 & 0.24 & 0.27 & 0.12 & 0.12 & \\
\hline NO & -0.08 & -0.05 & 0.15 & 0.00 & -0.46 & -0.40 & -0.0 \\
\hline NC & -0.19 & -0.20 & -0.05 & -0.22 & -0.39 & -0.39 & \\
\hline $\mathrm{CDE}$ & 0.07 & -0.10 & 0.06 & 0.03 & -0.36 & -0.36 & \\
\hline US & 0.06 & -0.16 & 0.15 & 0.07 & -0.21 & -0.47 & L - 1.0 \\
\hline & & & & & & & \\
\hline
\end{tabular}

C

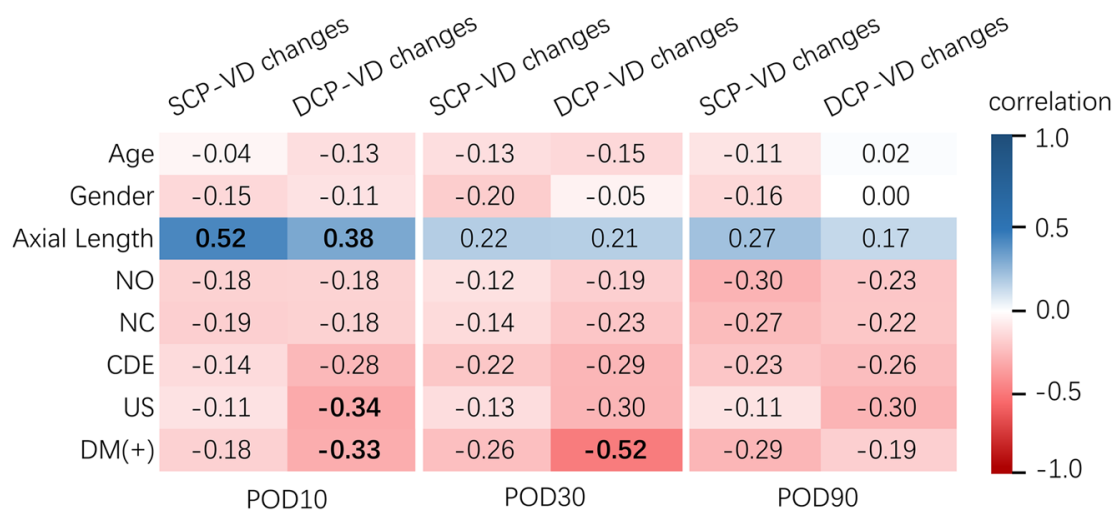

Fig. 4 The correlation matrix plot for the association between vessel density changes and baseline clinical characteristics in (a) diabetic patients $(n=$ 21), b non-diabetic controls $(n=21)$, and $\mathbf{c}$ all study subjects $(n=42)$. Statistically significant correlations are shown in bold. (POD = postoperative day; $\mathrm{SCP}=$ superficial capillary plexus; $\mathrm{DCP}=$ deep capillary plexus; $\mathrm{VD}=$ vessel density; $\mathrm{NO}=$ nuclear opalescence; $\mathrm{NC}=$ nuclear color; $\mathrm{CDE}=\mathrm{cumulative}$ dissipated energy; US = ultrasound total time; DM = diabetes mellitus; HbA1c = Hemoglobin A1c; NPDR = nonproliferative diabetic retinopathy)

subclinical macular thickening without apparent macular edema. Second, the CDE during cataract surgery was low and postoperative inflammatory reaction was mild. Third, patients received nonsteroidal anti-inflammatory eye drops in addition to steroid eye drops, which had been reported to be more effective than steroid only in preventing PCME [21] and might also play a role in influencing the pattern of macular microvascular changes in response to phacoemulsification surgery.

In correlation analysis, we found that the presence of diabetes is the key factor associated with postoperative DCP-VD reduction. In addition, surgical trauma and glycemic control are important add-on factors influencing DCP-VD changes in diabetic patients. There has been 
evidence that diabetes alone does not impair recovery from cataract surgery [22]. Our results suggested that the presence of diabetes is an independent factor associated with DCP-VD reduction after cataract surgery. Poor glycemic control is a well-recognized risk factor for developing PCME in diabetic patients [23, 24]. Consistent with previous reports, our results reinforced the importance of overall glycemic control in preventing surgical related macular changes. As opposed to previous studies which did not identify surgical trauma as important risk factors for developing PCME [24], this study detected a significant correlation between DCP-VD reduction and $\mathrm{CDE}$ in diabetic patients, probably due to the homogeneity of phacoemulsification surgeries performed by one single surgeon. Insulin dependence was likely to be associated with DCP-VD reduction at POD30 $(r=0.382, P=$ 0.087 ), but the association did not reach statistically significant, possibly due to the small sample size. Previous study indicated an increased risk of developing PCME in diabetic patients with pre-existing diabetic retinopathy [25]. In this study, the presence of baseline NPDR failed to show significant association with microvascular changes, probably because the number of subjects with baseline NPDR was quite limited. Interestingly, in the diabetic group, the DCP-VD reduction at POD10 was likely to be correlated with $\mathrm{CDE}(r=0.374, P=0.095)$ and US $(r=0.411, P=0.064)$, but not with HbA1c concentration $(r=0.272, P=0.23)$, suggesting that microvascular changes were likely to be affected by surgical trauma in the early postoperative period and then influenced by the combined effect of surgical trauma and glycemic control level in the later recovery stage.

The strengths of our study were the prospective study design, longitudinal follow-up, and a high-quality data acquisition. We acknowledge several limitations in our study. First, the number of included subjects was limited. We did not include enough patients with baseline NPDR. Our results could not be readily generalized to diabetic patients with pre-existing diabetic macular edema or proliferative diabetic retinopathy. Second, we only evaluated parafoveal vessel density with a $3 \times 3 \mathrm{~mm}$ field of view. The changes in perifoveal vessel density and the peripheral retina remained unknown. Third, we did not analyze the skeleton density changes. Fourth, we did not perform correlation analysis with visual outcome because the microvascular changes were subtle and the best corrected visual acuity of almost all patients reached $20 / 20$ at POD90.

\section{Conclusions}

Diabetic patients undergoing phacoemulsification surgery exhibited a transient mild reduction in vessel density of DCP at 1 month after surgery, which was correlated with postoperative macular thickening. Poor glycemic control and increased surgical trauma were found to be associated with the degree of DCP-VD reduction. Our findings offer new insights into the complex pathophysiological process of phacoemulsification surgery in diabetic patients and support the value of OCTA metrics in the management of postoperative changes in individuals with diabetes.

\section{Abbreviations \\ ANCOVA: Analysis of covariance; ANOVA: Analysis of variance; CDE: Cumulative dissipated energy; DCP: Deep capillary plexus; HbA1c: Hemoglobin A1c; OCTA: Optical coherence tomography angiography; NC: Nuclear color; NO: Nuclear opalescence; NPDR: Nonproliferative diabetic retinopathy; PCME: Pseudophakic cystoid macular edema; POD: Postoperative day; SCP: Superficial capillary plexus; US: Ultrasound total time; VD: Vessel density}

\section{Acknowledgements}

The authors acknowledge Dr. Yanlong Li for his statistical support.

\section{Authors' contributions}

$Z W, E W$, and $Y C$ designed the study. ZW performed all surgeries. ZW and EW collected and analyzed all data. ZW, EW, and YC interpreted the data and results. ZW and EW drafted the manuscript. YC reviewed the manuscript. All authors read and approved the final manuscript.

\section{Authors' information}

Null.

\section{Funding}

The study was supported by the Non-Profit Central Research Institute Fund of Chinese Academy of Medical Sciences (Grant No. 2018PT32029). The funding organization had no role in the design or conduct of this research.

\section{Availability of data and materials}

The datasets used and/or analysed during the current study are available from the corresponding author on reasonable request.

\section{Ethics approval and consent to participate}

The study was in compliance with the tenets of the Declaration of Helsinki. The Ethics Committee of Peking Union Medical College Hospital approved the study protocol. All participants gave written informed consent.

\section{Consent for publication}

Not applicable.

\section{Competing interests}

The authors declare that they have no competing interests.

Received: 9 June 2020 Accepted: 10 August 2020

Published online: 17 August 2020

\section{References}

1. Chu CJ, Johnston RL, Buscombe C, et al. Risk factors and incidence of macular edema after cataract surgery: a database study of 81984 eyes. Ophthalmology. 2016;123:316-23.

2. Bellocq D, Mathis T, Voirin N, et al. Incidence of Irvine Gass syndrome after phacoemulsification with spectral-domain optical coherence tomography. Ocul Immunol Inflamm. 2019;27:1224-31.

3. Samara WA, Shahlaee A, Adam MK, et al. Quantification of diabetic macular ischemia using optical coherence tomography angiography and its relationship with visual acuity. Ophthalmology. 2017;124:235-44.

4. Fawzi AA, Fayed AE, Linsenmeier RA, et al. Improved macular capillary flow on optical coherence tomography angiography after panretinal photocoagulation for proliferative diabetic retinopathy. Am J Ophthalmol. 2019:206:217-27.

5. Zhou Y, Zhou M, Wang $Y$, et al. Short-term changes in retinal vasculature and layer thickness after phacoemulsification surgery. Curr Eye Res. 2020:45: $31-7$ 
6. Pilotto E, Leonardi F, Stefanon G, et al. Early retinal and choroidal OCT and OCT angiography signs of inflammation after uncomplicated cataract surgery. Br J Ophthalmol. 2019;103:1001-7.

7. Zhao Z, Wen W, Jiang C, et al. Changes in macular vasculature after uncomplicated phacoemulsification surgery: optical coherence tomography angiography study. J Cataract Refract Surg. 2018:44:453-8.

8. Chylack LT Jr, Wolfe JK, Singer DM, et al. The Lens opacities classification system III. The longitudinal study of cataract study group. Arch Ophthalmol. 1993;111:831-6.

9. Wilkinson CP, Ferris FL 3rd, Klein RE, et al. Proposed international clinical diabetic retinopathy and diabetic macular edema disease severity scales. Ophthalmology. 2003;110:1677-82.

10. Yu S, Frueh BE, Steinmair D, et al. Cataract significantly influences quantitative measurements on swept-source optical coherence tomography angiography imaging. PLoS One. 2018;13:e0204501.

11. Bambo MP, Garcia-Martin E, Otin S, et al. Influence of cataract surgery on repeatability and measurements of spectral domain optical coherence tomography. Br J Ophthalmol. 2014;98:52-8.

12. Moore J, Bagley S, Ireland G, et al. Three dimensional analysis of microaneurysms in the human diabetic retina. J Anat. 1999;194:89-100.

13. Chen $\mathrm{Q}, \mathrm{Ma} \mathrm{Q}, \mathrm{Wu}$ C, et al. Macular vascular fractal dimension in the deep capillary layer as an early indicator of microvascular loss for retinopathy in type 2 diabetic patients. Invest Ophthalmol Vis Sci. 2017;58:3785-94.

14. Nesper PL, Roberts PK, Onishi AC, et al. Quantifying microvascular abnormalities with increasing severity of diabetic retinopathy using optical coherence tomography angiography. Invest Ophthalmol Vis Sci. 2017:58: Bio307-15.

15. Sun Z, Tang F, Wong $\mathrm{R}$, et al. OCT angiography metrics predict progression of diabetic retinopathy and development of diabetic macular edema: a prospective study. Ophthalmology. 2019;126:1675-84.

16. Hsiao CC, Yang CM, Yang CH, et al. Correlations between visual acuity and macular microvasculature quantified with optical coherence tomography angiography in diabetic macular oedema. Eye (Lond). 2019;34:1-9.

17. Sacconi R, Corbelli E, Carnevali A, et al. Optical coherence tomography angiography in pseudophakic cystoid macular oedema compared to diabetic macular oedema: qualitative and quantitative evaluation of retinal vasculature. Br J Ophthalmol. 2018;102:1684-90.

18. Chetrit M, Bonnin S, Mane V, et al. Acute pseudophakic cystoid macular edema imaged by optical coherence tomography angiography. Retina. 2018;38:2073-80.

19. Mane V, Dupas B, Gaudric A, et al. Correlation between cystoid spaces in chronic diabetic macular edema and capillary nonperfusion deteced by optical coherence tomography angiography. Retina. 2016;36(Suppl 1):S102-10.

20. Degenring RF, Vey S, Kamppeter B, et al. Effect of uncomplicated phacoemulsification on the central retina in diabetic and non-diabetic subjects. Graefes Arch Clin Exp Ophthalmol. 2007;245:18-23.

21. Laursen SB, Erichsen $\mathrm{JH}$, Holm LM, et al. Prevention of macular edema in patients with diabetes after cataract surgery. J Cataract Refract Surg. 2019; 45:854-69.

22. Danni R, Taipale C, Ilveskoski L, et al. Diabetes alone does not impair recovery from uneventful cataract surgery. Am J Ophthalmol. 2019;198:37-44.

23. Ylinen $\mathrm{P}$, Laine I, Lindholm JM, et al. Poor glycemic control as a risk factor for pseudophakic cystoid macular edema in patients with diabetes. J Cataract Refract Surg. 2017;43:1376-82.

24. Yang J, Cai L, Sun Z, et al. Risk factors for and diagnosis of pseudophakic cystoid macular edema after cataract surgery in diabetic patients. J Cataract Refract Surg. 2017:43:207-14

25. Samanta A, Kumar P, Machhua S, et al. Incidence of cystoid macular oedema in diabetic patients after phacoemulsification and free radical link to its pathogenesis. Br J Ophthalmol. 2014;98:1266-72.

\section{Publisher's Note}

Springer Nature remains neutral with regard to jurisdictional claims in published maps and institutional affiliations.

\section{Ready to submit your research? Choose BMC and benefit from:}

- fast, convenient online submission

- thorough peer review by experienced researchers in your field

- rapid publication on acceptance

- support for research data, including large and complex data types

- gold Open Access which fosters wider collaboration and increased citations

- maximum visibility for your research: over $100 \mathrm{M}$ website views per year

At BMC, research is always in progress.

Learn more biomedcentral.com/submissions 